\title{
GPIS HAVING COEFFICIENTS IN UTUMI QUOTIENT RINGS
}

\author{
CHEN-LIAN CHUANG
}

\author{
(Communicated by Donald S. Passman)
}

\begin{abstract}
Let $R$ be a prime ring and let $U$ be its Utumi quotient ring. We prove the following: (1) If $R$ satisfies a GPI having all its coefficients in $U$, then $R$ satisfies a GPI having all its coefficients in $R$. (2) $R$ and $U$ satisfy the same GPIs having their coefficients in $U$.
\end{abstract}

The main purpose of this paper is to generalize the two main theorems on generalized polynomial identities (GPIs) in [7] to their full generality. Our improvement is in two respects:

(1) The coefficients of the generalized polynomial identities are allowed to lie in the Utumi quotient rings instead of in the Martindale quotient rings.

(2) The assumption that the generalized polynomial identity is multilinear and homogeneous is removed. Our theorems hold for any arbitrary generalized polynomial identity.

Results in this generality seem to be interesting and should be useful elsewhere.

In what follows, $R$ is always a prime associative ring, not necessarily with identity 1. Let $U_{R}$ be the maximal rational extension of $R_{R}$ (as right $R$-modules) (see the definition and Theorem 6 on p. 59 [2]). Since $R$ is left faithful (p. 67 [2]), there is a natural ring operation on $U$ which induces the module operation $U \times R \rightarrow U$ (Proposition F on p. 68 [2]). $U$ endowed with the natural ring operation is an overring of $R$ and is called the Utumi quotient ring of $R$.

The Utumi quotient ring of $R$ can also be characterized axiomatically as follows: A right ideal $\rho$ of $R$ is said to be rational if and only if $R_{R}$ is a rational extension of $\rho_{R}$ (rational right ideals are also called dense right ideals in [6]). The Utumi quotient ring of $R$ is a ring $U$ satisfying the following axioms:

(1) $R$ is a subring of $U$.

(2) For each $a \in U$, there exists a rational right ideal $\rho$ of $R$ such that $a \rho \subseteq R$.

(3) If $a \in U$ and $a \rho=0$ for some rational right ideal $\rho$ of $R$, then $a=0$.

(4) For any rational right ideal $\rho$ and for any right $R$-module homomorphism $\phi: \rho_{R} \rightarrow R_{R}$, there exists $a \in U$ such that $\phi(r)=a r$ for all $r \in \rho$.

For a prime ring $R$, a nonzero two-sided ideal is obviously a rational right ideal of $R$. In the above axioms, if we consider only nonzero two-sided ideals instead of rational right ideals, then we obtain the Martindale quotient ring, which we denote by $Q$ (see [8] for the definition and [5] for the axiomatic formulation). $Q$ can be

Received by the editors June 29, 1987.

1980 Mathematics Subject Classification (1985 Revision). Primary 16A38; Secondary 16A08, $16 \mathrm{~A} 12$.

Key words and phrases. Generalized polynomial identity, Utumi quotient ring, Martindale quotient ring. 
naturally regarded as a subring of $U$ and can be characterized as follows: For $a \in U$, $a \in Q$ if and only if $a I \subseteq R$ for some nonzero two-sided ideal $I$ of $R$.

Note that both $U$ and $Q$ are prime (for $U$, see p. 74 [2]; the assumption there on the singular right ideal is unnecessary). Also observe that the center of $U$, denoted by $C$, coincides with the center of $Q$. $C$ is called the extended centroid of $R$.

In what follows, by submodules, we always mean right $R$-submodules of $U$. By a rational submodule $M$, we mean a right $R$-submodule $M$ of $U$ such that $U_{R}$ is a rational extension of $M_{R}$.

The following theorem is our main tool in dealing with generalized polynomials with coefficients in $U$ :

THEOREM 1. Assume that $R$ is a prime ring and $U$ is its Utumi quotient ring. Let $N$ be a rational submodule of $U$ and let $u_{1}, \ldots, u_{n} \in U$ be $C$-linearly independent. Then there exists $a \in N$ such that $u_{1} a, \ldots, u_{n} a \in N$ and such that $u_{1} a, \ldots, u_{n} a$ are still $C$-linearly independent.

We start our proof with

LEMMA 1. If $R$ has a nonzero right ideal $\rho$ which is finite dimensional over $C$, then $R$ itself is finite dimensional over $C$.

Proof. Suppose that $\operatorname{dim}_{C} \rho C=m<\infty$. Since $R$ acts faithfully on $\rho C$ by right multiplication, $R$ embeds in $M_{m}(C)$, the ring of $m \times m$ matrices over $C$. So $R$ is finite dimensional.

The following lemma, which is a slight modification of Lemma 1 [1], is the key step to our proof.

LEMMA 2. Let $V$ and $W$ be two vector spaces over a field $F$ and let $T_{1}, \ldots, T_{r}$, be $F$-linearly independent linear transformations of $V$ into $W$. Let $B$ be an additive subgroup of $V$ such that $F B=V$. Then for any finite dimensional subspace $W_{0}$ of $W$, either there exists $v \in B$ such that $T_{1} v, \ldots, T_{r} v$ are linearly independent module $W_{0}$, or there exists $S=\sum_{i=1}^{r} \alpha_{i} T_{i} \neq 0$, where $\alpha_{i} \in F$, which is of finite rank.

Lemma $1[1]$ is the same as Lemma 2 except where $B$ is assumed to be the whole $V$. For the proof of Lemma 2 above, we simply replace each occurrence of $V$ by $B$ in Amitsur's proof of Lemma 1 [1] (and also $U, U_{0}$ there by $W, W_{0}$ here for notation difference). We omit the details for brevity.

PROOF OF THEOREM 1. Let $N$ be the given rational submodule of $U$. Set $M=N \cap u_{1}^{-1} N \cap u_{2}^{-1} N \cap \cdots \cap u_{n}^{-1} N$, where $u_{i}^{-1} N=\left\{u \in U: u_{i} u \in N\right\} . M$, an intersection of finitely many rational submodules, is itself a rational submodule of $U$. Via left multiplication, we may regard $u_{1}, u_{2}, \ldots, u_{n}$ as $C$-linear transformations from the $C$-vector space $M C$ into the $C$-vector space $N C$. If there exists $a \in M$ such that $u_{1} a, u_{2} a, \ldots, u_{n} a$ are $C$-linearly independent, then we are done. Otherwise, by Lemma 2 , there exist $\alpha_{1}, \alpha_{2}, \ldots, \alpha_{n} \in C$ such that $u=\alpha_{1} u_{1}+\alpha_{2} u_{2}+\cdots+\alpha_{n} u_{n}$ is a nonzero finite rank linear transformation on $M C$ (via left multiplication). Set $L=M \cap u^{-1} R$. Then $L$ is a rational submodule of $M$ such that $0 \neq u L \subseteq R$. Since $u$ is of finite rank on $M, u L$ must also be finite $C$-dimensional. Thus $R$ possesses a nonzero, finite $C$-dimensional right ideal $u L$. By Lemma $1, R$ is also finite $C$ dimensional and hence must be a PI-ring. Set $\rho=N \cap R$, which is a rational right ideal of $R$. For a prime PI-ring $R, U, Q$ and $R C$ are all equal to the localization of $\rho$ 
at its center $Z(\rho)$ by Theorem 2 (p. 57 [4]). So we can find a common denominator $\alpha \in Z(\rho)$ such that $\alpha u_{1}, \alpha u_{2}, \ldots, \alpha u_{n} \in \rho$. Since $Z(\rho) \subseteq C, \alpha u_{1}, \alpha u_{2}, \ldots, \alpha u_{n}$ are obviously $C$-linearly independent.

Let $X=\left\{x_{1}, x_{2}, \ldots\right\}$, the countable set consisting of the noncommuting indeterminates $x_{1}, x_{2}, \ldots$ Let $C\{X\}$ be the free algebra over $C$ in the set $X$. Consider $U\{X\}=U *_{C} C\{X\}$, the free product over $C$ of $U$ and $C\{X\}$. Elements of $U\{X\}$ are called generalized polynomials. By a nontrivial generalized polynomial, we mean a nonzero element of $U\{X\}$. An element $m \in U\{X\}$ of the form $m=q_{0} y_{1} q_{1} y_{2} q_{2} \cdots y_{n} q_{n}$, where $\left\{q_{0}, \ldots, q_{n}\right\} \subseteq U$ and $\left\{y_{1}, \ldots, y_{n}\right\} \subseteq X$, is called a monomial. $q_{0}, \ldots, q_{n}$ are called the coefficients of $m$. Each $f \in U\{X\}$ can be represented as a finite sum of monomials. Such representation is certainly not unique. For a given representation of $f$ as a sum of monomials, the coefficients of each monomial occurring in the representation are called the coefficients of $f$ in the given representation. For a subset $V$ of $U, f \in U\{X\}$ is called a $V$-generalized polynomial if and only if $f$ has a representation with all of its coefficients in $V$. Hence every generalized polynomial is trivially a $U$-generalized polynomial.

Let $B$ be a set of $C$-independent vectors of $U$. By a $B$-monomial, we mean a monomial of the form $u_{0} y_{1} u_{1} y_{2} u_{2} \cdots y_{n} u_{n}$, where $\left\{u_{0}, \ldots, u_{n}\right\} \subseteq B$ and where $\left\{y_{1}, y_{2}, \ldots, y_{n}\right\} \subseteq X$. Let $V=B C$, the $C$-subspace spanned by $B$. Then any $V$-generalized polynomial $f$ can be written in the form $\sum \alpha_{i} m_{i}$, where $\alpha_{i} \in C$ and where $m_{i}$ are $B$-monomials, in the following manner: First, fix a representation of $f$ with all of its coefficients in $V$ and express each coefficient of the given representation as a linear combination of elements in $B$. Then substitute these linear combinations into the representation of $f$ and expand the resulting expression using the distributive law. Finally, we collect similar terms to get our desired form.

It is also obvious that such representation of a given $f$ in terms of $B$-monomials is unique. This is actually equivalent to saying that $B$-monomials form a basis for the $C$-vector space consisting of all $B C$-generalized polynomials. If $B$ is chosen to be a basis of $U$ over $C$, then $B$-monomials span the whole $U\{X\}$.

The uniqueness of representation in terms of $B$-monomials gives a practical criterion to decide whether a given generalized polynomial $f$ is trivial or not: Pick a basis $B$ for the $C$-subspace spanned by the coefficients of a given representation of $f$. Express $f$ as a linear combination of $B$-monomials in the way explained above. Let us say $f=\sum \alpha_{i} m_{i}$, where $\alpha_{i} \in C$ and $m_{i}$ are $B$-monomials. Then $f$ is trivial if and only if $\alpha_{i}=0$ for each $i$. This simple criterion will be used in the proofs below to assure the nontriviality of a given generalized polynomial.

Our next objective is to prove the following two theorems, which generalize respectively Theorems 1 and 2 of [7].

THEOREM 2. Assume that $R$ is a prime ring and $U$ is its Utumi quotient ring. For any rational submodule $M$ of $U$, the GPIs satisfied by $M$ are the same as the GPIs satisfied by $U$.

THEOREM 3. Assume that $R$ is a prime ring and $U$ is its Utumi quotient ring. Let $M$ and $N$ be two rational submodules of $U$. If $M$ satisfies a GPI, then $M$ satisfies a $N$-GPI.

Instead of rational submodules of $U$, Theorem 1 [7] and Theorem 2 [7] are formulated for $R$-subbimodules of $Q$. As remarked in [7], for any $R$-subbimodule 
$T$ of $Q$ (or of $U$ as well), $T \cap R$ is a nonzero two-sided ideal of $R$ and hence $T$ itself must be a rational submodule of $U$. Thus our theorems above actually generalize theorems in [7].

The following lemma is crucial.

LEMMA 3. Let $N$ be a rational submodule of $U$ and let $f\left(x_{1}, \ldots, x_{n}\right)$ be a nontrivial generalized polynomial. Then there exists $a \in N$ such that $f\left(a x_{1}, \cdots, a x_{n}\right) a$ is a nontrivial $N$-generalized polynomial.

Proof. Choose a basis $B$ for the $C$-subspace spanned by the coefficients of a representation of $f$ and write $f=\sum_{i=1}^{s} \alpha_{i} m_{i}$, where $\alpha_{i} \in C \backslash\{0\}$ and where $m_{i}$ are distinct $B$-monomials. Note that $B$ is a finite set.

Set $M=\left(\bigcap_{i=1}^{s} \alpha_{i}^{-1} N\right) \cap N . M$ is also a rational submodule of $U$. By Theorem 1 , there exists $a \in M$ such that $\{u a: u \in B\}$ is a $C$-linearly independent subset of $M$.

Consider a $B$-monomial $m\left(y_{1}, \ldots, y_{k}\right)=u_{0} y_{1} u_{1} y_{2} u_{2} \cdots y_{k} u_{k}$, where $\left\{u_{0}, \ldots, u_{k}\right\}$ $\subseteq B$ and $\left\{y_{1}, \ldots, y_{k}\right\} \subseteq X$. Then

$$
m\left(a y_{1}, \ldots, a y_{k}\right) a=\left(u_{0} a\right) y_{1}\left(u_{1} a\right) y_{2}\left(u_{2} a\right) \cdots y_{k}\left(u_{k} a\right)
$$

where $u_{0} a, u_{1} a, \ldots, u_{k} a \in\{u a: u \in B\}$. Set $B^{\prime}=\{u a: u \in B\}$. By our choice of $a, B^{\prime}$ is an independent set of $M$. Hence $m\left(a y_{1}, \ldots, a y_{k}\right) a$ is a $B^{\prime}$-monomial. Also, if $\alpha$ is one of $\alpha_{1}, \ldots, \alpha_{s}$, then

$$
\alpha m\left(a y_{1}, \ldots, a y_{k}\right) a=\left(\alpha u_{0} a\right) y_{1}\left(u_{1} a\right) y_{2}\left(u_{2} a\right) \cdots y_{k}\left(u_{k} a\right) .
$$

By our choice of $a, u_{0} a, u_{1} a, \ldots, u_{k} a \in M \subseteq N$. By the definition of $M, \alpha u_{0} a \in$ $\alpha M \subseteq N$. Hence $\alpha m\left(a y_{1}, \ldots, a y_{k}\right) a$ is an $N$-generalized polynomial.

Now, consider $f\left(a x_{1}, \ldots, a x_{n}\right) a=\sum_{i=1}^{s} \alpha_{i} m_{i}\left(a x_{1}, \ldots, a x_{n}\right) a$. By the result of the previous paragraph, each $m_{i}\left(a x_{1}, \ldots, a x_{n}\right) a$ is a $B^{\prime}$-monomial. Hence $f\left(a x_{1}, \ldots, a x_{n}\right) a$ is nontrivial. Again, by the result of the previous paragraph, each

$$
\alpha_{i} m_{i}\left(a x_{1}, \ldots, a x_{n}\right) a
$$

is an $N$-generalized polynomial and hence so is $f\left(a x_{1}, \ldots, a x_{n}\right) a$, as desired.

We need the following special case of Theorem 2 .

LEMMA 4. Let $M$ be a rational submodule of $U$. If $M$ satisfies a nontrivial $G P I$, then $R$ satisfies a nontrivial $R$-GPI.

ProOF. Let $f\left(x_{1}, \ldots, x_{n}\right)=0$ be a nontrivial GPI satisfied by $M$. Set $\rho=$ $M \cap R . \quad \rho$ is a rational right ideal of $R$. By Lemma 3, there exists $a \in \rho$ such that $f\left(a x_{1}, \ldots, a x_{n}\right) a$ is a nontrivial $\rho$-generalized polynomial. For $r_{1}, \ldots, r_{n} \in R$, $a r_{1}, \ldots, a r_{n} \in \rho R \subseteq \rho \subseteq M$. Hence $f\left(a r_{1}, \ldots, a r_{n}\right) a=0$. So $R$ satisfies the nontrivial $\rho$-GPI $f\left(a x_{1}, \ldots, a x_{n}\right) a=0$.

PROOF OF THEOREM 2. Let $M$ be a rational submodule of $U$. It is obvious that any GPIs satisfied by $U$ are also satisfied by $M$. So we show the converse.

If every GPI satisfied by $M$ is trivial, then there is nothing to prove. So we assume that $M$ satisfies a nontrivial GPI. By Lemma $4, R$ satisfies a $R$-GPI. By the main results in [8], the central closure $S(=R C)$ of $R$ contains a minimal idempotent $e$ such that $e S e$ is a finite dimensional division algebra over $C$. Note that the socle of $S$ is nonzero. By Proposition 7 (p. 98 [6]) and its proof, the Utumi 
quotient ring of $S$ is canonically isomorphic to $\operatorname{Hom}(S e, S e)_{e S e}$. Also, under this canonical isomorphism, $S$ is realized via left multiplication as a dense subring of $\operatorname{Hom}(S e, S e)_{e S e}$. From now on, we identify each $s \in S$ with the left multiplication on $S e$ by $s$. Then the Utumi quotient ring of $S$ is $\operatorname{Hom}(S e, S e)_{e S e}$. Since $S$ is a rational extension of $R$, the Utumi quotient ring of $S$ coincides with the Utumi quotient ring of $R$. So we have $U=\operatorname{Hom}(S e, S e)_{e S e}$.

Let $\rho=M \cap R$ and let $\sigma$ denote the socle of $S$. Since any GPI is continuous with respect to the finite topology on $\operatorname{Hom}(S e, S e)_{e S e}$ (see p. 248 [3] for the definition) and since $\sigma$ is dense in $\operatorname{Hom}(S e, S e)_{e S e}$ with respect to the finite topology, it suffices to show that each GPI satisfied by $\rho$ is also satisfied by $\sigma$.

First, suppose that $C$ is finite. Then there exists a rational right ideal $\rho^{\prime}$ of $R$ such that $\alpha \rho^{\prime} \subseteq R$ for all $\alpha \in C$. Consider $\rho \rho^{\prime}$. Since $\rho \rho^{\prime}$ is a rational right ideal of $R, \rho \rho^{\prime} C$ is a rational right ideal of $S$ and hence $\rho \rho^{\prime} C \supseteq \sigma$. But $\rho \rho^{\prime} C \subseteq \rho R \subseteq \rho$. So $\rho \supseteq \sigma$. Thus any GPI vanishing on $\rho$ also vanishes on $\sigma$ as is desired.

Now we assume that $C$ is infinite. Let $f$ be a GPI of $\rho$. We proceed by induction on the height of $f$ to show that $f$ vanishes on $U$. Pick sufficiently but finitely many distinct $\alpha \in C$. Let $\rho^{\prime}$ be a rational right ideal of $R$ such that $\alpha \rho^{\prime} \subseteq \rho$ for all those $\alpha$ we have picked. Replace each indeterminate $x$ in $f$ by $\alpha x$ for these $\alpha$. Then the resulting GPIs vanish on $\rho^{\prime}$. So, using the Vandermonde determinant argument, we can solve for the homogeneous parts of $f$. So each homogeneous part of $f$ vanishes on $\rho^{\prime}$. It suffices to show that each homogeneous part of $f$ vanishes on $U$. Note that the height of each homogeneous part of $f$ is less than or equal to that of $f$. Replacing $f$ by one of its homogeneous parts and $\rho$ by $\rho^{\prime}$, we may assume from the start that $f$ is homogeneous in each indeterminate it involves.

Assume that the height of $f$ is zero. Then, since $f$ is homogeneous, $f$ must be multilinear. By the multilinearity, $f$ vanishes on $\rho C$. But $\rho C$, a rational right ideal of $S$, must include $\sigma$. So $f$ vanishes on $\sigma$ and hence on $U$ as desired. So we assume that the height of $f$ is larger than 0 . As the induction hypothesis, we also assume that the assertion holds for any GPI whose height is less than that of $f$.

Let $x$ be an indeterminate involved in $f$. For this moment, we suppress all indeterminates other than $x$ and write $f=f(x)$ for simplicity of notations. Consider $g(x, y)=f(x+y)-f(x)-f(y)$, where $y$ is a new indeterminate not occurring in $f$. Since $g$ is obviously of less height than $f$ and since $g$ vanishes on $\rho, g$ must vanish $U$ by our induction hypothesis. So $f(x+y)=f(x)+f(y)$ for $x, y \in U$. We have thus shown that $f$ is additive on $U$ with respect to each indeterminate it involves.

Now write $f=f\left(x_{1}, \ldots, x_{n}\right)$, where $x_{1}, \ldots, x_{n}$ are all the indeterminates which $f$ involves. Set $x_{i}=\sum_{j} r_{j}^{(i)} \alpha_{j}^{(i)}$, where $r_{j}^{(i)} \in \rho$ and $\alpha_{j}^{(i)} \in C$. Using the additivity of $f$ on $U$, we compute

$$
\begin{aligned}
f\left(x_{1}, \ldots, x_{n}\right) & =f\left(\sum_{j} r_{j}^{(1)} \alpha_{j}^{(1)}, \ldots, \sum_{j} r_{j}^{(n)} \alpha_{j}^{(n)}\right) \\
& =\sum_{j_{1}, \ldots, j_{n}} f\left(r_{j_{1}}^{(1)} \alpha_{j_{1}}^{(1)}, \ldots, r_{j_{n}}^{(n)} \alpha_{j_{n}}^{(n)}\right) \\
& =\sum_{j_{1}, \ldots, j_{n}}\left(\alpha_{j_{1}}^{(1)}\right)^{h_{1}} \cdots\left(\alpha_{j_{n}}^{(n)}\right)^{h_{n}} f\left(r_{j_{1}}^{(1)}, \ldots, r_{j_{n}}^{(n)}\right)
\end{aligned}
$$


where $h_{i}$ is the $x_{i}$-degree of $f(i=1, \ldots, n)$. Since $r_{j_{i}}^{(i)} \in \rho, f\left(r_{j_{1}}^{(1)}, \ldots, r_{j_{n}}^{(n)}\right)=0$. Hence $f\left(x_{1}, \ldots, x_{n}\right)=0$. But $x_{i}=\sum_{j} r_{j}^{(i)} \alpha_{j}^{(i)}$ are typical elements of $\rho C$. Since $\rho C$, a rational right ideal of $R C$, must include $\sigma, f$ vanishes on $\sigma$ and hence on $U$ as is desired.

ProOF OF THEOREM 3 . Let $M, N$ be two given rational submodules of $U$. In view of Theorem 2, we may assume that $M=U$. Suppose that $f\left(x_{1}, \ldots, x_{n}\right)=0$ is a GPI of $U$. Then by Lemma 3 , there exists $a \in N$ such that $f\left(a x_{1}, \ldots, a x_{n}\right) a$ is a nontrivial $N$-generalized polynomial. Obviously, $f\left(a x_{1}, \ldots, a x_{n}\right) a=0$ is also a $N$-GPI for $U$.

ACKNOWLEDGEMENT. The author would like to thank the referee for suggesting the simple proof of Lemma 1 which we present here.

\section{REFERENCES}

1. S. A. Amitsur, Generalized polynomial identities and pivotal monomials, Trans. Amer. Math. Soc. 114 (1965), 210-226.

2. C. Faith, Lectures on injective modules and quotient rings, Lecture Notes in Math., vol. 49, Springer-Verlag, Berlin and New York, 1967.

3. N. Jacobson, Lectures in abstract algebra. II, Van Nostrand, New York, 1953.

4. __ PI-Algebras, An Introduction, Lecture Notes in Math., vol 441, Springer-Verlag, Berlin and New York, 1975.

5. V. K. Kharchenko, Differential identities of prime rings, Algebra i Logika 17 (1976), 220-238.

6. J. Lambek, Lectures on rings and modules, Blaisdell, Waltham, Mass., 1966.

7. C. Lanski, A note on GPIs and their coefficients, Proc. Amer. Math. Soc. 98 (1986), 17-19.

8. W. S. Martindale III, Prime rings satisfying a generalized polynomial identity, J. Algebra 12 (1969), 576-584.

9. N. H. McCoy, The theory of rings, Macmillan, New York, 1964.

Department of Mathematics, National Taiwan University, Taipei, Taiwan 10764, REPUBLIC OF CHINA 